\title{
The Sabadell score is an independent predictor of five-year outcome after critical care discharge
}

Ben Morton ( $\nabla$ ben.morton@lstmed.ac.uk)

Liverpool School of Tropical Medicine https://orcid.org/0000-0002-6164-2854

Victoria Penston

University Hospital Aintree

Phillip McHale

University of Liverpool

Daniel Hungerford

University of Liverpool

Ged Dempsey

University Hospital Aintree

\section{Research}

Keywords: Critical care, Survival analysis, Rehabilitation, death

Posted Date: December 24th, 2019

DOI: https://doi.org/10.21203/rs.2.19576/v1

License: @) (7) This work is licensed under a Creative Commons Attribution 4.0 International License. Read Full License 


\section{Abstract}

Background: Critical care survivors frequently suffer persistent morbidity and increased risk of mortality compared to the general population. However, there are no standardised tools to identify at-risk patients to target potential interventions. Our aim was to establish whether the "Sabadell score", a simple tool applied upon critical care discharge, is an independent predictor of five-year mortality.

Methods: Prospective observational cohort study of adults admitted to a mixed critical care unit at Aintree University Hospital NHS Foundation Trust, Liverpool, United Kingdom. Sabadell score applied to all patients from September 2011 to December 2017. Primary outcome: five-year mortality assessed using a multivariable flexible parametric survival analysis adjusted for demographics, and clinically relevant covariates. Primary outcome: Adults discharged alive following critical care admission.

Results: There were 5954 patients with a minimum of 18 months follow-up. Mean age was $59.5(\mathrm{SD} \pm 17)$ and $57.1 \%$ were male. Patients were categorised into Sabadell scores, zero (38.4\%), one (47.9\%), two (10.5\%) and three (3.1\%). Adjusted hazard ratios for mortality were 2.09 (C.I. 1.85-2.36), 3.95 (C.I. 3.39-4.60) and 21.04 (C.I. 17.24-25.68) respectively. Sabadell score three predicted 99.9\%, 99.5\%, 98.5\% and $87.4 \%$ mortality at five years for patients ${ }^{3} 80$ (aHR 3.37 ), 60 79 (aHR 2.52), 40-59 (aHR 2.03) and 16-39 respectively. A Sabadell score of two predicted $71.0 \%, 52.7 \%, 44.8 \%$ and $23.7 \%$ mortality at five years for these age categories.

Conclusions : Sabadell score is an independent predictor of five-year survival after critical care discharge. These findings could be used to guide provision of increased support for patients after critical care discharge and/or informed discussions with patients and relatives about dying to ascertain their future wishes.

\section{Introduction}

There is increasing recognition of the need to quantify longer term outcomes for patients after critical care admission. Critical care survivors suffer continued and significant healthcare burden, (1-3) and decreased longer-term survival compared to the general population $(4,5)$. Such tools could be used to guide interventions to improve outcomes for at-risk patients (6). In addition, there is increasing recognition of the need to hold prospective informed discussions with patients (and their families) at increased risk of dying to ascertain their wishes and plan for future care (7). However, there are currently no validated tools to inform provision of increased resources nor to inform the initiation of discussions about death at the point of critical care discharge.

Critical care scoring systems frequently focus on outcome prediction for the hospital admission episode rather than long-term survival (2,8-10). Further, these tools can be complex and labour intensive $(5,11)$, with variable discriminative ability $(12)$. The Sabadell Score was developed and validated as a simplified tool based on the judgement of the attending critical care physician to predict the likelihood of in-hospital mortality after critical care discharge (13, 14). However, the longer-term discriminative ability of this tool for deaths before and after hospital discharge has not been determined

The aim of this study was to investigate the utility of the Sabadell Score as an independent predictor of long-term outcomes after discharge from a UK critical care unit. If validated, this simple scoring system could facilitate improved communication to guide prospective and informed care decisions with patients and their relatives at the point of critical care discharge.

\section{Methods}

\section{Setting and participants}

A prospective cohort of adult patients admitted to a 23-bed critical care unit at the multi-site Liverpool Universities Hospital NHS Foundation Trust, UK between 1st September 2011 and 31st December 2017. The critical care unit is a mixed medical and surgical unit for adult patients requiring level two or level three care (15) with a maximum patient to nurse ratio of 2:1. The hospital is a regional referral centre for trauma, hepato-biliary and head and neck surgery. Adult patients (aged $\geq 16$ from all specialties are admitted, with the exception of cardiothoracic and plastic surgery. The unit is staffed by two consultant intensivists during day-time hours and has resident consultant (attending) cover out of hours. Within the hospital, there is a medical emergency team, inclusive of a critical care representative and an outreach service who routinely review patients after critical care discharge. Patients discharged alive from the critical care unit were included in the study. Patients transferred to another critical care unit and those with insufficient data to allow analysis were excluded (Fig. 1). For patients with multiple critical care admissions, only the index admission was incorporated into the analysis. Survival data for all patients was last updated in July 2019.

\section{Data Collection}

The data were recorded prospectively on a database as part of the UK intensive care national audit project (Intensive Care National Audit and Research Centre (ICNARC) case mix programme (16)). These included: age, sex, postcode (zipcode), primary diagnosis, specialty (medical / surgical), nature of admission (elective / emergency), APACHE II score and length of ICU stay. For the study, patients were each assigned a Sabadell score by the consultant intensivist, based on their experience of treating the patient at critical care discharge (13): Sabadell 0: good prognosis; Sabadell 1: poor long term diagnosis; Sabadell 2: poor short term diagnosis and Sabadell 3: death expected during current hospital admission. The allocated score was not documented in the patient's case notes and blinded to the ward team taking over the care of the patient. We differentiated critical care readmission data according to hospital admission status (same or separate admission). Readmission within the same hospital episode were recorded for individual patients (potential marker of poor prognosis) within the analysis. 
On discharge from our unit, all patients routinely undergo direct hand over to the relevant ward team, including discussion of ongoing issues and specific plans for subsequent management. Ceilings of care were documented in the case notes independently from the allocation of the Sabadell score, if indicated. Hospital electronic records (Medway System C Healthcare Ltd - used for hospital episode statistics nationally) were reviewed in July 2019 to determine the long-term outcomes of the patients. The Hospital Episode Statistics are routinely collected for all admissions to NHS hospitals and contain reliable, comprehensive patient information used widely in the UK (17). Therefore, all patients were subject to a minimum of eighteen-month follow up.

To assess the effect of socioeconomic deprivation on outcome, the Index of Multiple Deprivation (IMD) was used (18). The Index of Multiple Deprivation (IMD) is based on the home postcode (zipcode) mapped to Lower Super Output Area (LSOA). LSOAs are geographic areas designed to report small area statistics (mean population 1500) in England and Wales. The IMD score is derived from weighted scores from seven domains; income, employment and health deprivation, disability, barriers to housing and services, crime, living environment and education \& skill deprivation. LSOA IMD scores are further categorised into deciles with decile one being the most deprived and decile ten the least (19).

\section{Research Approval}

The Research and Development Department of Aintree University Hospital sponsored this study and ethical approval was granted, following registration with the Integrated Research Application System (IRAS) website (17/NE/0007). Due to the observational nature of the study, consent was not required from the patients. Anonymity was maintained throughout the data handling.

\section{Statistical Analysis}

Patient characteristics (gender, age, IMD score, APACHE II score, medical/surgical diagnosis, emergency/elective admission, readmissions, hospital length of stay, year of admission and mortality) were analysed according to Sabadell score. Patient survival was further explored according to Sabadell score for patient survival at the point of hospital discharge and 30 days, 90 day, six months, one year, two years, three years and five years post critical care discharge. Variables were expressed as frequency and percentages for categorical variables and median with interquartile ranges for numerical variables. To identify factors independently associated with survival at hospital discharge (dichotomous dependent variable), we conducted univariable logistical regression analysis with the above described patient characteristics (independent variables) to produce crude odds ratio. These variables were all subsequently included within the multivariable logistical regression analysis to produce adjusted odds ratios. The relationship between Sabadell score and five-year survival was initially explored with Cox-regression analysis. However, multiple variables (Sabadell score, elective vs. emergency, medical vs. surgical and APACHE II score) were found to violate proportional hazard assumptions (Table S1). Therefore, a flexible parametric survival model was fitted using the STATA "stmp2" command (20) to produce hazard ratios. The most parsimonious model was identified at six internal splines using the Bayesian information criterion method (Table S2). Statistical significance was accepted at the $5 \%$ level. Sensitivity analyses were performed including and excluding in-hospital mortality for univariable (Table S3) and multivariable (Table S4) analysis. Data were analysed using Stata V15.1 (StataCorp, Stata Statistical Software: Release 15, College Station, Texas, USA) and figures produced using R V.3.5.1 (R Development Core Team, Vienna, Austria).

\section{Results}

During the study period, there were 8374 patients admitted to the ICU. When discharges direct to other hospitals, readmissions, critical care non-survivors, patients lost to follow up and those with non-English postcodes were removed 5954 patients were included in the final analysis (Fig. 1). Patients with nonEnglish postcodes were more likely to be elective surgical admissions with lower Sabadell scores and improved survival, reflective of the hospital status as a tertiary referral centre (Table S5). Table two shows the baseline characteristics of the study population split by Sabadell score. Males were responsible for $57.1 \%$ of admissions and mean age was 59.5 (SD \pm 17 ) years. A Sabadell score of zero was assigned to $38.4 \%$ (2287/5953) of the population, one assigned to $47.9 \%$ (2854/5953), two assigned to 10.5\% (629/5953) and three to $3.1 \%(183 / 5953)$ respectively. We observed that the mean age, mean APACHE II score, proportion of medical versus surgical admissions, proportion of emergency admissions and the proportion of hospital mortality were highest in patients assigned a Sabadell score of three (Table 1). 
Table 1

\begin{tabular}{|c|c|c|c|c|c|c|c|}
\hline \multirow[t]{2}{*}{ Variable } & & \multirow{2}{*}{$\begin{array}{l}\text { Overall } n= \\
5954\end{array}$} & \multicolumn{4}{|c|}{ Sabadell score } & \multirow[t]{2}{*}{$P$ value } \\
\hline & & & $0(n=2287)$ & $1(n=2854)$ & $2(n=629)$ & $3(n=183)$ & \\
\hline Gender (male) & $\mathrm{n}(\%)$ & $3396(57.1)$ & $1316(57.5)$ & $1636(57.3)$ & $347(55.1)$ & $97(53.0)$ & $0.481 x$ \\
\hline Age & Mean (SD) & $59.5(17.0)$ & $50.4(17.7)$ & $64.5(13.6)$ & $67.1(14.2)$ & $70.4(13.6)$ & $\begin{array}{l}< \\
0.001^{\mathrm{a}}\end{array}$ \\
\hline APACHE II & Mean (SD) & $15(5.8)$ & $12.95(2.1)$ & $15.56(5.2)$ & $18.0(6.0)$ & $20.6(5.6)$ & $0.571^{a}$ \\
\hline Medical admission & $\mathrm{n}(\%)$ & $2317(38.9)$ & $878(38.4)$ & $974(34.1)$ & $352(56.0)$ & $113(61.7)$ & $\begin{array}{l}< \\
0.001 x\end{array}$ \\
\hline Emergency admission & n (\%) & $4081(68.5)$ & $1622(70.9)$ & $1735(60.8)$ & $545(86.6)$ & $179(97.8)$ & $\begin{array}{l}<.001 x \\
0.00\end{array}$ \\
\hline $\begin{array}{l}\text { Readmission in same hospital } \\
\text { stay }\end{array}$ & $\mathrm{n}(\%)$ & $227(3.8)$ & $65(2.8)$ & $126(4.4)$ & $35(5.6)$ & $1(0.5)$ & $\begin{array}{l}< \\
0.001 x\end{array}$ \\
\hline LSOA IMD score & $\begin{array}{l}\text { Median } \\
\text { (IQR) }\end{array}$ & $\begin{array}{l}37.6(18.4- \\
57.2)\end{array}$ & $\begin{array}{l}39.2(19.2- \\
57.2)\end{array}$ & $\begin{array}{l}35.8(17.5- \\
57.1)\end{array}$ & $\begin{array}{l}39.5(19.5- \\
57.2)\end{array}$ & $\begin{array}{l}40.7(19.5- \\
56.1)\end{array}$ & $0.037^{\beta}$ \\
\hline
\end{tabular}

Table three shows survival data for the patient cohort. There was at least 18 months follow up data for all patients. Complete follow up data was available for $94.3 \%(5616 / 5953)$ at two years, $82.2 \%(4897 / 5953)$ at three years and $61.8 \%(3676 / 5953)$ at five years respectively. Overall mortality was $4.9 \%$ at 30 days, $8.1 \%$ at 90 days, $17.2 \%$ at one year, $26.6 \%$ at two years, $36.3 \%$ at three years and $57.5 \%$ at five years. For patients with a Sabadell score of two, $50.3 \%$ had died at two years (Table 2). In agreement with previous work $(13,14)$, logistic regression analysis demonstrated that the Sabadell score was an independent predictor of hospital mortality in both univariable and multivariable analyses. The adjusted odds ratios for Sabadell scores 1, 2 and 3 were 5.08 (C.I. $2.61-$ 9.91), 22.06 (C.I. 11.15-43.68) and 278.06 (C.I. 134.33-575.59) respectively (Fig. 2 and Table S6).

Table 2

\begin{tabular}{|llllll|}
\hline Mortality at given time $(\mathbf{n})$ & Overall frequency $\mathbf{n}(\%)$ & \multicolumn{4}{l}{ Mortality according to Sabadell score $\mathbf{n}(\%)$} \\
\cline { 3 - 6 } & & $\mathbf{0}$ & $\mathbf{1}$ & $\mathbf{2}$ & $\mathbf{3}$ \\
\hline Hospital discharge (5953) & $334(5.6)$ & $10(0.4)$ & $92(3.2)$ & $102(16.2)$ & $130(71.0)$ \\
\hline 30 Day (5953) & $289(4.9)$ & $5(0.2)$ & $65(2.3)$ & $87(13.8)$ & $132(72.1)$ \\
\hline 90 Day (5953) & $481(8.1)$ & $30(1.3)$ & $158(5.5)$ & $148(23.5)$ & $145(79.2)$ \\
\hline Year (5953) & $978(17.2)$ & $118(5.2)$ & $470(16.5)$ & $232(37.7)$ & $158(87.3)$ \\
\hline 2 Years (5616) & $1494(26.6)$ & $226(10.6)$ & $793(29.5)$ & $311(50.3)$ & $164(90.6)$ \\
\hline 3 Years (4897) & $1777(36.3)$ & $303(17.2)$ & $955(40.0)$ & $350(60.9)$ & $169(94.9)$ \\
\hline 5 Years (3676) & $2155(57.5)$ & $375(33.8)$ & $1170(62.3)$ & $398(77.1)$ & $172(98.3)$ \\
\hline
\end{tabular}

To explore the relationship between Sabadell score and five-year mortality we used a flexible parametric survival model. We included hospital deaths in this model because, clinically, the Sabadell score was assigned at the point of critical discharge and this is when the opportunity for discussion with patients and/or next of kin could occur. All a priori selected variables (Sabadell score, age, gender, elective vs. emergency admission, medical vs. surgical admission, LSOA score, APACHE II score, need for readmission, hospital length of stay and year of admission) were included in both univariable and multivariable analyses. The adjusted hazard ratios for Sabadell scores 1, 2 and 3 were 2.15 (C.I. 1.90-2.43), 4.17 (C.I. 3.57-4.88) and 23.60 (C.I. 19.15-29.07) respectively (Table S4). Using this model, we predicted five-year survival for Sabadell score and age category (16-39, 40-60, 60-80 and $\geq 80$ as the variables with the greatest magnitude of effect. Predictions were adjusted for the remaining co-variates within the multivariable model (Fig. 3 ). Based on this model more than $50 \%$ of patients allocated a Sabadell score of three would be expected to die within two months from critical care discharge ( $\geq 80)$, three months (60-79), five months (40-59) and 13 months (16-39) respectively. An additional data file provides numerical detail for model predictions [see additional data file 1]. For patients allocated a Sabadell score of two, more than $50 \%$ would be expected to die within 26 months ( $\geq 80)$ and 40 months (60-79) respectively (Supplementary data).

For sensitivity analysis, we removed patients who died during their index hospital admission following critical care discharge and applied the flexible parametric survival model. The most parsimonious model was identified at two internal splines using the Bayesian information criterion method (data not shown)., This demonstrated that Sabadell scores one (aHR 2.00, C.I. 1.77-2.27), two (aHR 3.25 (C.I. 2.76-3.84) and three (aHR 7.12,C.I. 5.11-9.92) predicted death for patients who survived their index hospital admission (Tables S3 and S4). Survival predictions based on this model are displayed in Figure S1. An additional data file provides numerical detail for model predictions [see additional data file 2]

\section{Discussion}


Our results demonstrate that the Sabadell score is an independent predictor of five-year survival after critical care discharge. Sabadell scores of two and three predicted poor patient survival for patients discharged from critical care both in-hospital and for patients who survive their hospital episode. This instrument could potentially be used to guide informed discussions with patients (and/or their relatives) about dying to ascertain their future wishes. Further multicentre studies are required to validate our findings for critical care patients and potentially, patients admitted under other specialties.

There is increasing recognition of the need "to empower doctors, patients and carers to make shared decisions about care and treatment that balance duration and quality of life" (7). In the UK nearly half of all deaths occur in hospital (21). Poor communication in the final stages of life is a frequently cited source of complaint. Subsequently, the UK Royal College of Physicians have proposed a number of recommendations for medical professionals to initiate timely and honest conversations with patients about their future (7). Episodes of illness that require critical care admission increasingly result from acute exacerbations of chronic disease rather than a single curable event. Patients who survive a critical illness episode frequently suffer significant and persistent morbidity $(1,2$, 22).

The point of discharge from critical to ward-based care represents an opportunity to intervene for high-risk patients and/or guide discussion between professionals and their relatives about death to ascertain their wishes. Our study demonstrates that the Sabadell score, a subjective prediction of longer-term patient outcome, has strong potential to guide initiation of these discussions. We observed this effect for both patients who died in hospital after their critical care discharge and for those who survived their index admission. The observed effect for patients allocated a Sabadell score of three was lower for hospital survivors (aHR 7.13 vs. 23.60). As Sabadell score three patients are expected to die in hospital, this is an expected finding. Importantly, patients who survived hospital admission despite allocation of this score still had a persistently higher risk of death compared to patients allocated Sabadell scores zero, one and two (Table S4).

Our study confirms previous findings that the Sabadell score is strongly predictive of in-hospital mortality $(13,14)$. This suggests that the tool may be of use in identifying patients at risk of physiological deterioration once discharged to the ward, as described previously (14). For example, patients with a Sabadell score of two could be managed with an enhanced level of support with an aim to prevent deterioration and death in hospital and improve survival in the community. In contrast to the study by Soliman et al, we found that the Sabadell score was strongly predictive of outcomes at one year post critical care discharge (9). We noted that critical care doctors from our unit were pessimistic with allocation of Sabadell scores with patient survival frequently living beyond their allocated score. However, on a population level, the Sabadell score could be a very useful tool to signpost "at-risk" patients to guide rehabilitation interventions post critical care discharge and/or determine if they are willing to engage with end-of-life discussion and begin the process of advanced care planning.

Despite data from a single centre only, we have accumulated a large cohort with comprehensive follow up for at least 18 months after critical care discharge. We have produced long term data on survival, but could not collect data to assess of quality of life, an important outcome measure for critical care survivors (23). Previous concerns regarding the Sabadell scoring system relate to the concept of a self-fulfilling prophecy, in other words, allocating a score to any particular patient may affect decision making in relation to their future management and that relying on subjective opinions alone may not be acceptable for this reason. (24) In the present study, the ward team were blinded to the patients Sabadell score, therefore, care received should be independent of the score. In the situation whereby a patient was deemed not suitable for further admission to intensive care, this decision was made before allocating the score.

We found that in-hospital mortality was $5.6 \%$ after critical care discharge, in keeping with previously published data $(14,25,26)$. Mortality one year following critical care discharge was $17.2 \%$, also in keeping with previous studies $(3,4,9,22,26)$. There appears to be more limited data on mortality at five years, however, the finding of an overall mortality of $64.5 \%$ appears to be higher than recent data published by Lone (32\%) (22). Contrary to finding from other studies $(27,28)$, we did not find a relationship between socioeconomic deprivation and longer term outcome. This is potentially explained by the distribution of socioeconomic deprivation in our cohort: $40.8 \%$ of patients were from the lowest income decile (Table S7).

\section{Conclusions}

We recommend further multicentre studies to assess the external validity of our findings in critical care patients. Clinicians should also investigate the validity of this tool in patients outside of critical care. This simple tool, based on a clinician's prior knowledge of how a patient has responded to treatment on critical care, has now been validated as a predictor of hospital survival in multiple studies. Clinicians may consider using the tool to direct rehabilitation and therapies to prevent subsequent deterioration following ward discharge. Risk stratification for critical care outreach teams to direct care delivery has the potential to improve outcomes and could inform successful delivery of future interventions. Talking about dying is potentially distressing for patients, carers and care givers. We suggest that with further validation, the Sabadell score could be used to prospectively direct such discussions.

\section{Declarations}

Ethical approval and consent to participate

This study was reviewed and approved by the North East NHS Research Ethics Committee (Reference: 17/NE/0007). As this study was purely observational in nature and no identifiable patient details were used, the ethics committee waived the need for individual participant consent.

\section{Consent for publication}

Not applicable

Availability of data and materials 
The datasets used and/or analysed during the current study are available from the corresponding authors on reasonable request.

Competing interests

The authors declare no competing interests in relation to this work

Funding

No funding was received to support this study.

Author contributions

GD and BM jointly conceived the study ideas and design of the project. GD and VP acquired data. BM, PM and DH performed statistical analysis. BM, VP, PM, $\mathrm{DH}$ and GD interpreted the analysis. BM, VP, PM, DH and GD jointly drafted the manuscript. All authors gave final approval for this version to be published.

\section{Acknowledgements}

The authors would like to thank the critical care consultants who completed the Sabadell Scores for this study. Listed in alphabetical order: Tim Astles, Lyndsay Ayres, Tristan Cope, Briggite Dupont, Chris Grant, Raj Nagaraja, Eoghan O'Callaghan, Robert Parker, Lauren Ruff, Vinoth Sankar, Sashika Selladurai, Jane Snell, Jenny Rodrigues and Simon Rogers. The authors would also like to thank Professor Graham Devereux for his critical appraisal of the manuscript. The authors declare no funding or conflicts of interest in relation to this work.

\section{References}

1. Needham DM, Davidson J, Cohen $\mathrm{H}$, Hopkins RO, Weinert C, Wunsch $\mathrm{H}$, et al. Improving long-term outcomes after discharge from intensive care unit: report from a stakeholders' conference. Crit Care Med. 2012;40(2):502-9.

2. Garland A, Olafson K, Ramsey CD, Yogendran M, Fransoo R. A population-based observational study of intensive care unit-related outcomes. With emphasis on post-hospital outcomes. Ann Am Thorac Soc. 2015;12(2):202-8.

3. Hill AD, Fowler RA, Pinto R, Herridge MS, Cuthbertson BH, Scales DC. Long-term outcomes and healthcare utilization following critical illness-a population-based study. Crit Care. 2016;20:76.

4. Gayat E, Cariou A, Deye N, Vieillard-Baron A, Jaber S, Damoisel C, et al. Determinants of long-term outcome in ICU survivors: results from the FROG-ICU study. Crit Care. 2018;22(1):8.

5. Hoffman RL, Saucier J, Dasani S, Collins T, Holena DN, Fitzpatrick M, et al. Development and implementation of a risk identification tool to facilitate critical care transitions for high-risk surgical patients. Int J Qual Health Care. 2017;29(3):412-9.

6. Walsh TS, Salisbury LG, Merriweather JL, Boyd JA, Griffith DM, Huby G, et al. Increased Hospital-Based Physical Rehabilitation and Information Provision After Intensive Care Unit Discharge: The RECOVER Randomized Clinical Trial. JAMA Intern Med. 2015;175(6):901-10.

7. Royal College of Physicians UK: Talking about dying: How to begin honest conversations about what lies ahead https://www.rcplondon.ac.uk/projects/outputs/talking-about-dying-how-begin-honest-conversations-about-what-lies-ahead [

8. Fika S, Nanas S, Baltopoulos G, Charitidou E, Myrianthefs P. A novel mortality prediction model for the current population in an adult intensive care unit. Heart Lung. 2018;47(1):10-5.

9. Soliman IW, Cremer OL, de Lange DW, Slooter AJC, van Delden JHJM, van Dijk D, et al. The ability of intensive care unit physicians to estimate long-term prognosis in survivors of critical illness. J Crit Care. 2018;43:148-55.

10. Ferrando-Vivas P, Jones A, Rowan KM, Harrison DA. Development and validation of the new ICNARC model for prediction of acute hospital mortality in adult critical care. J Crit Care. 2017;38:335-9.

11. Wernly B, Lichtenauer M, Vellinga NAR, Boerma EC, Ince C, Kelm M, et al. Blood urea nitrogen (BUN) independently predicts mortality in critically ill patients admitted to ICU: A multicenter study. Clin Hemorheol Microcirc. 2018;69(1-2):123-31.

12. Hosein FS, Bobrovitz N, Berthelot S, Zygun D, Ghali WA, Stelfox HT. A systematic review of tools for predicting severe adverse events following patient discharge from intensive care units. Crit Care. 2013;17(3):R102.

13. Fernandez R, Baigorri F, Navarro G, Artigas A. A modified McCabe score for stratification of patients after intensive care unit discharge: the Sabadell score. Crit Care. 2006;10(6):R179.

14. Fernandez R, Serrano JM, Umaran I, Abizanda R, Carrillo A, Lopez-Pueyo MJ, et al. Ward mortality after ICU discharge: a multicenter validation of the Sabadell score. Intensive Care Med. 2010;36(7):1196-201.

15. Department of Health UK. Comprehensive Critical Care: a review of adult critical care services https://webarchive.nationalarchives.gov.uk/+/http://www.dh.gov.uk/en/Publicationsandstatistics/Publications/PublicationsPolicyAndGuidance/DH_400 [

16. ICNARC. National Audit Programme (NAP) 2019 [Available from: https://www.icnarc.org/Our-Audit/About.

17. Digital N. Hospital Episode Statistics (HES) 2019 [Available from: https://digital.nhs.uk/data-and-information/data-tools-and-services/dataservices/hospital-episode-statistics.

18. The English indices of deprivation. https://www.gov.uk/government/statistics/english-indices-of-deprivation-2015.

19. The Index of Multiple Deprivation 2015: A Liverpool analysis. https://liverpool.gov.uk/media/10001/1-imd-2015-executive-summary.pdf. 
20. Lambert P, Royston P. Further development of flexible parametric models for survival analysis. Stata J. 2009;9:265-90.

21. ONS. National Survey of Bereaved People (VOICES): England, 2015. Quality of care delivered in the last 3 months of life for adults who died in England: ONS; 2016 [Available from:

www.ons.gov.uk/peoplepopulationandcommunity/healthandsocialcare/healthcaresystem/bulletins/nationalsurveyofbereavedpeoplevoices/england2015

22. Lone NI, Gillies MA, Haddow C, Dobbie R, Rowan KM, Wild SH, et al. Five-Year Mortality and Hospital Costs Associated with Surviving Intensive Care. Am J Respir Crit Care Med. 2016;194(2):198-208.

23. Desai SV, Law TJ, Needham DM. Long-term complications of critical care. Crit Care Med. 2011;39(2):371-9.

24. Afessa B, Keegan MT. Predicting mortality in intensive care unit survivors using a subjective scoring system. Crit Care. 2007;11(1):109.

25. Gayat E, Cariou A, Deye N, Vieillard-Baron A, Jaber S, Damoisel C, et al. Determinants of long-term outcome in ICU survivors: results from the FROG-ICU study. Crit Care. 2018;22(1):8.

26. Meynaar IA, Van Den Boogaard M, Tangkau PL, Dawson L, Sleeswijk Visser S, Bakker J. Long-term survival after ICU treatment. Minerva Anestesiol. 2012;78(12):1324-32.

27. McHale P, Hungerford D, Taylor-Robinson D, Lawrence T, Astles T, Morton B. Socioeconomic status and 30-day mortality after minor and major trauma: A retrospective analysis of the Trauma Audit and Research Network (TARN) dataset for England. PLoS One. 2018;13(12):e0210226.

28. Hutchings A, Raine R, Brady A, Wildman M, Rowan K. Socioeconomic status and outcome from intensive care in England and Wales. Med Care. 2004;42(10):943-51.

\section{Figures}

\section{Figure 1}

Patients admitted to the Intensive Care Unit (ICU) during the study period

展

\section{Figure 2}

Adjusted and unadjusted odds ratios for in-hospital mortality after critical care discharge in our patient cohort. Figure displays unadjusted (black) and adjusted (red) odds ratios for our a priori selected variables within the analysis. Reference variables are described on the $y$-axis and assigned an odds ratio of

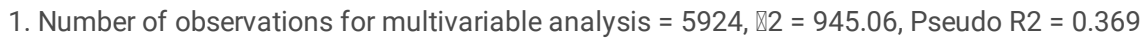



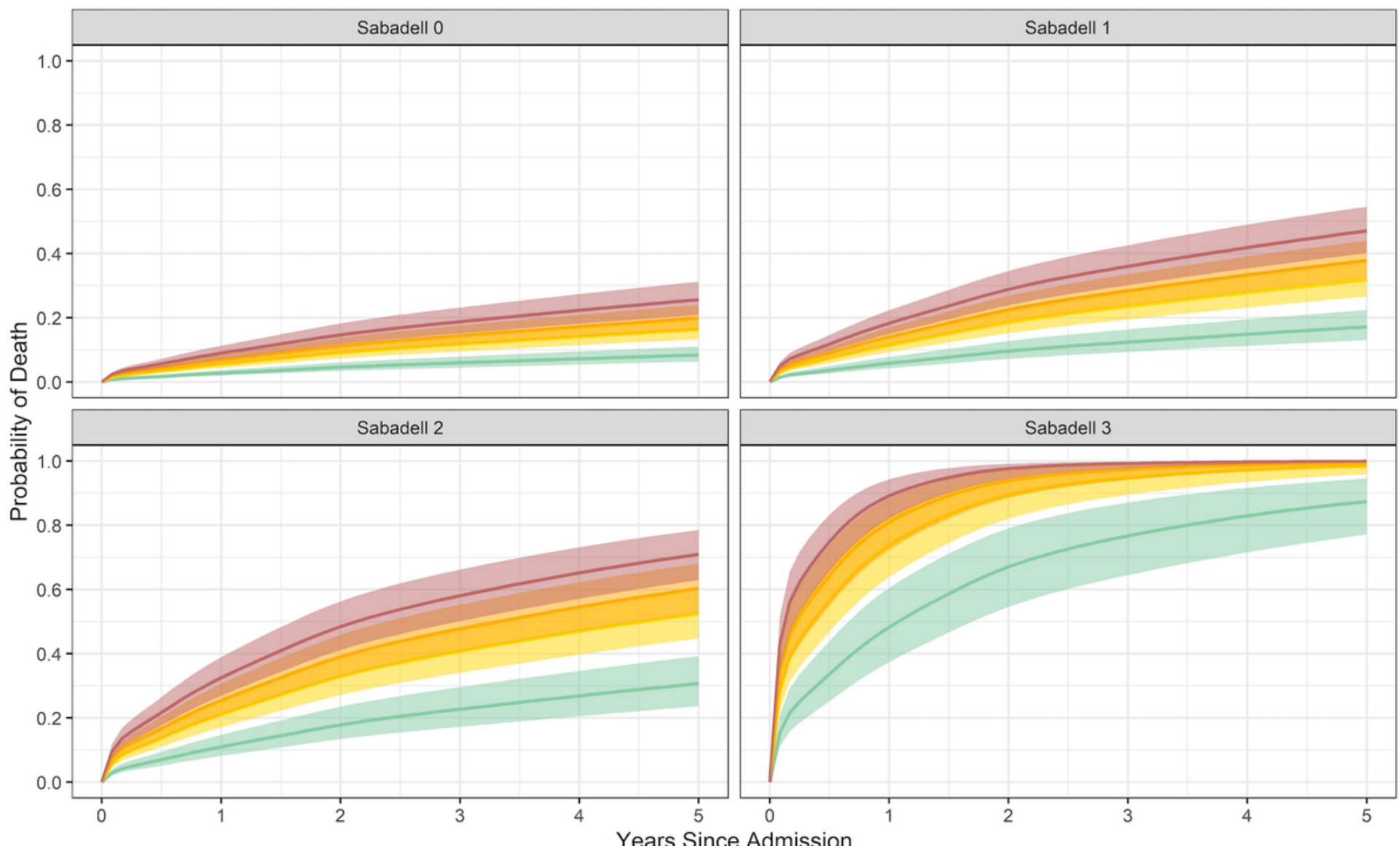

- $16-39-40-59-60-79-80+$

\section{Figure 3}

Survival predictions for Sabadell score and age category based on a flexible parametric survival model for five-year survival after critical care discharge. Each panel represents predicted survival by Sabadell score according to age category after adjustment for gender, APACHE II score, elective vs. emergency admission, medical vs. surgical admission, social deprivation score and need for readmission within the same spell. The flexible parametric survival model was fitted with six internal splines and includes 5924 individual patient observations.

\section{Supplementary Files}

This is a list of supplementary files associated with this preprint. Click to download.

- Mortalitypredictionsforalldeaths.csv

- Onlinesupplementarymaterial.docx

- Mortalitypredictionsfordeathsafterhospitalsurvival.csv 